\title{
A fiber optic buckle transducer for measurement of in vitro tendon strain
}

\author{
Paulo Roriz ${ }^{\mathrm{a} ; \mathrm{c}}$, António Ramos ${ }^{\mathrm{b}}$, Manuel B Marques ${ }^{\mathrm{c}}$, José A Simões ${ }^{\mathrm{b}, \mathrm{d}}$, Orlando Frazão ${ }^{\mathrm{c}}$ \\ ${ }^{a}$ University Institute of Maia (ISMAI), Av. Carlos Oliveira Campos, Castelo da Maia, 4475-690 \\ Maia, Portugal; ${ }^{b}$ Department of Mechanics, University of Aveiro, Campo Universitário de Santiago, \\ 3810-193 Aveiro, Portugal; INESC TEC, Centre for Applied Photonics (CAP), Rua do Campo \\ Alegre, 687, 4150-179 Porto, Portugal; ${ }^{\mathrm{d}}$ Escola Superior de Artes e Design (ESAD), Avenida \\ Calouste Gulbenkian, 4460-268 Senhora da Hora, Matosinhos, Portugal
}

\begin{abstract}
The purpose of the present study is to present a prototype of a fiber optic based buckle transducer suitable for measuring strain caused by stretching of a tendon. The device has an E-shape and its central arm is instrumented with a fiber Bragg grating (FBG) sensor. The tendon adjusts to the E-form in a fashion that when it is stretched the central arm bends causing a shift of the Bragg's wavelength $\left(\lambda_{B}\right)$ that is proportional to the amount of strain. This prototype is presented as an alternative to conventional strain gauge (SG) buckle transducers.
\end{abstract}

Keywords: Strain, Buckle transducers, Fiber optic sensors, Fiber Bragg grating, Tendon, Finite element analysis.

\section{INTRODUCTION}

The study ligament or tendon strain is a main topic in medicine and sports. Ligaments connect bone to bone. They resist stretch to assure the stability and congruency of a joint or a group of joints. Ligaments act like joint controllers of the range of motion. Tendons connect muscle to bone transferring to them the force generated during muscle contraction. Thus, studying the strain and forces acting in these tissues will contribute to understand some of their main functions. Usually, in situ strain measurement of tendons is measured with strain gauge (SG) based transducers. The buckle transducer, introduced by Salmons [1], has been the most used. The buckle can present several different shapes, such as a rectangular or oval form [2-5] or an E-form [6,7]. Buckle dimensions depend on tissue geometry [8]. Fiber optic sensors (FOSs) have a more recent history than SG but they seem very promising for biomechanics and biomedical applications $[9,10]$. In particular, fiber Bragg gratings (FBGs) are in good position to substitute or to be used as an alternative to SGs. Compared to them, they are smaller, easier to implement, minimally invasive, with lower risk of infection, highly accurate, well correlated, inexpensive and multiplexable [11]. FBG sensors have a linear response to axial strain and provide direct and absolute measurements [12].

\section{MATERIAL AND METHODS}

A PMMA E-form buckle transducer was manufactured and instrumented with a FBG with a Bragg wavelength $\left(\lambda_{B}\right)$ centered at $1541 \mathrm{~nm}$ and with a length of $5.0 \mathrm{~mm}$. The FBG was glued to the central arm of the buckle (Figure 1).

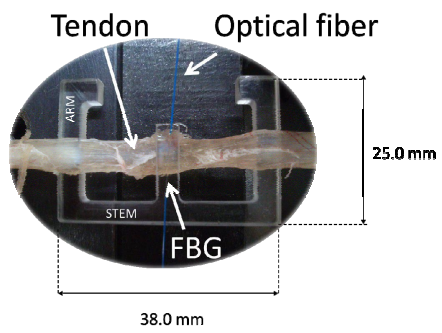

Figure1 - Schematic representation of the E-buckle sensor. The FBG was glued to the central arm.

24th International Conference on Optical Fibre Sensors, edited by Hypolito José Kalinowski, José Luís Fabris, Wojtek J. Bock, Proc. of SPIE Vol. 9634, 96342Q · ( 2015 SPIE CCC code: $0277-786 X / 15 / \$ 18 \cdot$ doi: $10.1117 / 12.2195249$ 
A fresh bovine tendon sample was used for strain measurements. As can be depicted from the previous figure when the tendon is stretched it causes the central arm to bend. The bending strain was measured in the compressed side of arm by a shift of $\lambda_{\mathrm{B}}$ using an optical spectrum analyzer. To minimize the temperature effect the experiment was performed at constant temperature $\left(\approx 20^{\circ}\right)$.

A linear translation stage was used to stretch the tendon (Figure 2). One end of the tendon was held fixed with a clamp and the other clamped to the moving platform of the translation stage. The length of the tendon between the clamps was $90.0 \mathrm{~mm}\left(\mathrm{~L}_{0}\right)$. A precision adjustment screw with a micrometer head (resolution of $1 \mu \mathrm{m}$ ) was used generate displacements of $10 \mu \mathrm{m}$ (step) up to $0.2 \mathrm{~mm}$.

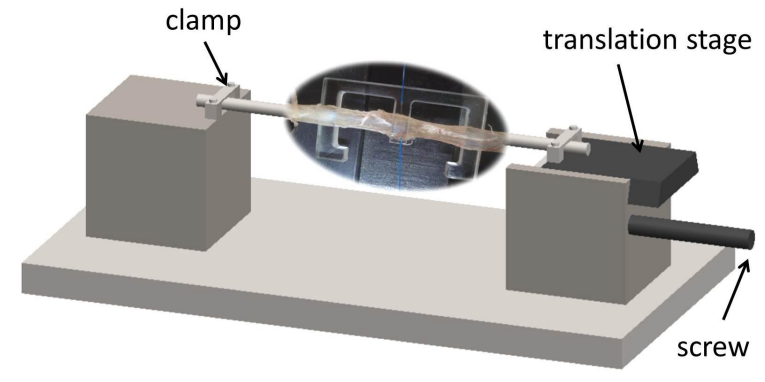

Figure 2 - Schematic drawing of the setup used to stretch the tendon.

Data from five repetitions was collected and tendon strain $(\mu \varepsilon)$ versus the average Bragg wavelength shift (nm) was plotted for analysis of buckle sensitivity.

Finite element analysis (FEA) was also performed to compare strain distribution in the buckle with strain measured by the FBG. Both, experimental and finite element models had the same geometry. The finite element model was constructed with 8 nodes hexahedral linear elements (figure 3). For PMMA a Young modulus of 3.0 GPa and 0.3 Poisson coefficients have been defined. The tendon was modeled as a uniform round corner rectangular section with dimensions similar to those of the experimental tendon $(5 \mathrm{~mm} \times 1.5 \mathrm{~mm}$ ) (figure 3$)$. A Young modulus of $2 \mathrm{GPa}$ and 0.45 Poisson coefficients have been defined for the tendon [13].

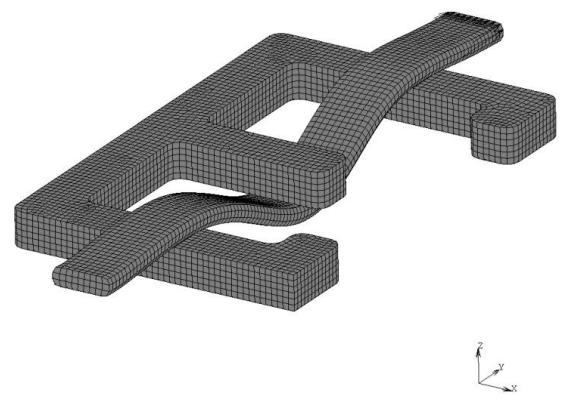

Figure 3 - CAD model of the E-form buckle transducer and tendon.

The boundary condition was an imposed displacement of $0.2 \mathrm{~mm}$ for the tendon in one side and considering no translation in buckle transducer in perpendicular direction of tendon imposed displacement. The contact between the tendon and the buckle was defined as touching with a friction coefficient of 0.01 .

\section{RESULTS AND DISCUSSION}

The results from five repetitions are plotted in figure 4. 


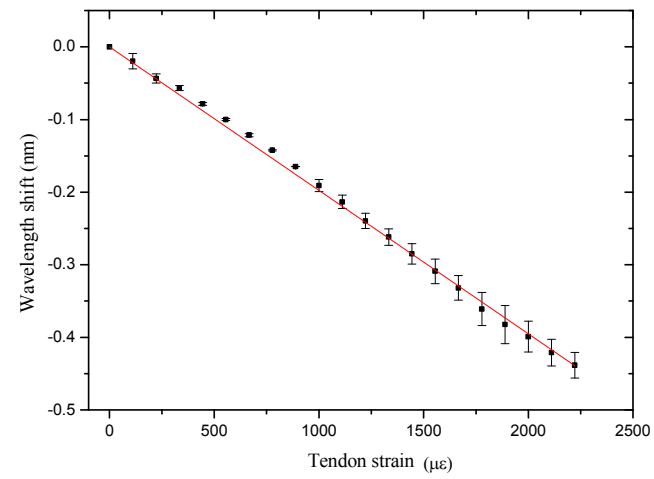

Figure 4 - Wavelength shift (nm) versus tendon strain $(\mu \varepsilon)$.

The $\lambda_{\mathrm{B}}$ shift for each displacement step was $0.020 \pm 0.005 \mathrm{~nm}$ (mean \pm standard deviation). The standard deviation was one order of magnitude less than the mean value. However as can be observed from the previous figure the error seems to increase with the applied strain.

In order to calculate the wavelength sensitivity to tendon strain a linear fit was performed (equation 1). The mean sensitivity obtained was $-0.2 \mathrm{pm} / \mu \varepsilon$.

$$
y(n m)=-1.97591 \times 10^{-4} \times x(\mu \varepsilon)
$$

In figure 5 the results of FEA for strain distribution along in the central arm of the buckle are plotted.

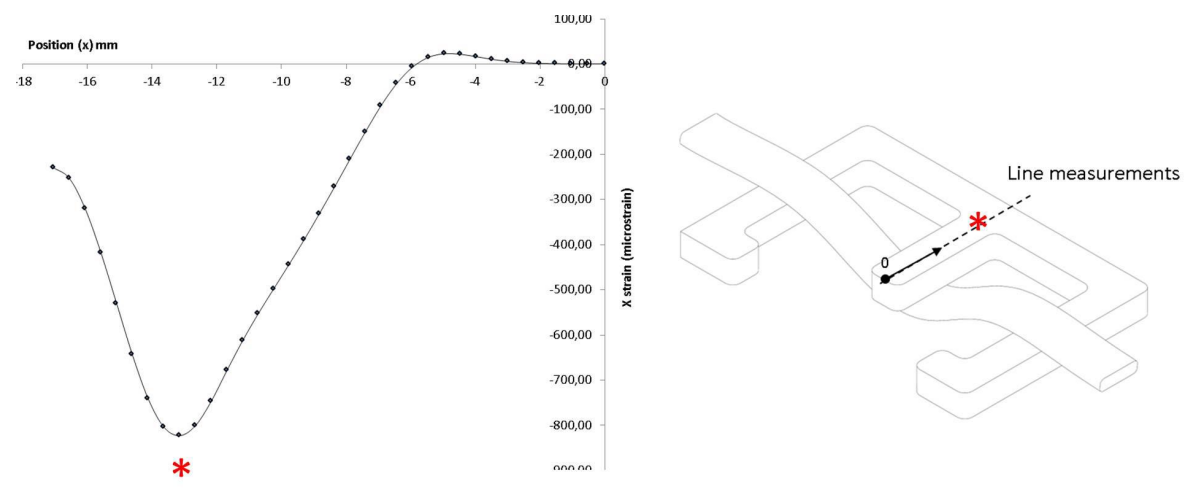

Figure 5 - Axial strain deformation in line

As expected, the maximum value of strain $(-822 \mu \varepsilon)$ for an imposed displacement of $0.2 \mathrm{~mm}$ was observed at the junction between the central arm and the stem of the buckle (13.15 mm from the origin of the line of measurement) (figure 5). For the same imposed displacement of $0.2 \mathrm{~mm}$ the average strain measured in the region of the FBG $(5 \mathrm{~mm}$ length) was $-327.60 \mu \varepsilon$. Using a conversion factor of $1.2 \mathrm{pm} / \mu \varepsilon$ a similar value $(-365.4 \mu \varepsilon)$ was obtained for the FBG. In the near future smaller and multiplexed FBGs will be used to better correlate experimental and FEA strain measurements along the central arm of the buckle.

\section{CONCLUSIONS}

A fiber optic based buckle transducer suitable for measuring tendon stretch was proposed. Future developments will include use of multiplexed FBGs, the implementation of new geometries and buckle materials, and sensor optimization for in situ and ex vivo measurements. 


\section{ACKNOWLEDGMENTS}

This work was supported by Project "NORTE-07-0124-FEDER-000058" which is financed by the North Portugal Regional Operational Programme (ON.2- O Novo Norte), under the National Strategic Reference Framework (NSRF), through the European Regional Development Fund (ERDF), and by national funds, through FCT.

\section{REFERENCES}

[1] Salmons, S., "Meeting Report," Proc 8th Int Conf Med Biol Eng, pp. 467-474 (1969).

[2] Lewis, J. L., Lew, W. D. and Schmidt, J., "A note on the application and evaluation of the buckle transducer for the knee ligament force measurement," J Biomech Eng-T ASME. 104(2), 125-128 (1982).

[3] An, K. N., Berglund, L., Cooney, W. P., Chao, E. Y. and Kovacevic, N., "Direct in vivo tendon force measurement system," J Biomech, 23(12), 1269-1271 (1990).

[4] Komi, P. V. "Relevance of in vivo force measurements to human biomechanics," J Biomech, 23(1), 23-34 (1990).

[5] Fukashiro, S., Komi, P. V., Järvinen, M. and Miyashita, M. "Comparison between the directly measured achilles tendon force and the tendon force calculated from the ankle joint moment during vertical jumps," Clin Biomech, 8 (1), 25-30 (1993).

[6] Walmsley, B., Hodgson, J. A. and Burke, R. E. "Forces produced by medial gastrocnemius and soleus muscles during locomotion in freely moving cats," J Neurophysiol, 41, 1203-1216, (1978).

[7] Komi, P. V.,Salonen, M., Järvinen, M. and O. Kokko, "In vivo registration of Achilles tendon forces in man. I. Methodological development," Int J Sports Med, 8(1), 3-8 (1987).

[8] Ravary, B. , Pourcelot, P., Bortolussi, C., Konieczka, S. and Crevier-Denoix, N. "Strain and force transducers used in human and veterinary tendon and ligament biomechanical studies," Clinical Biomech, 19(5), 433-447 (2004).

[9] Roriz, P., Ramos, A., Santos, J.L. and Simões, J. "Fiber optic intensity-modulated sensors: A review in biomechanics," Photonic Sensors, 2(4), 315-330 (2012).

[10] Roriz, P., Carvalho, L., Frazão, O., Santos, J. and Simões, J. A. "From conventional sensors to fibre optic sensors for strain and force measurements in biomechanics applications: A review," J. Biomech., 47(6), 1251-1261 (2014).

[11] Roriz, P., Frazão, O., Lobo-Ribeiro, A. Santos, J. and Simões, J. "Review of fiber optic pressure sensors for biomedical and biomechanical applications," J Biomed Optics, 18(5), 1-18 (2013).

[12] Othonos, A. "Fiber Bragg gratings," Rev Sci Instrum, 68(12), 4309-4341 (1997).

[13] Kongsgaard, M., Nielsen, C. H., Hegnsvad, S., Aagaard, P. and Magnusson, S. P. "Mechanical properties of the human Achilles tendon, in vivo," Clinical Biomech, 26(7), $772-777$ (2011).

*pjro@inesctec.pt; paulororiz@ismai.pt

Proc. of SPIE Vol. 9634 96342Q-4 